\title{
Familial combined cellular and humoral immune defect with multisystem granulomata
}

\author{
W. H. PERKS AND IAN S. PETHERAM \\ From Brompton Hospital, Fulham Road, London SW3 6HP, UK
}

\begin{abstract}
Perks, W. H., and Petheram, I. S. (1978). Thorax, 33, 101-105. Familial combined cellular and humoral immune defect with multisystem granulomata. A brother and sister presented with recurrent respiratory infections. Investigations showed combined cellular and humoral immune defects and non-caseating granulomata in lungs, liver, lymph nodes, and skin. Despite antimycobacterial chemotherapy and corticosteroids in the brother and the additional treatment of human immunoglobulin injections in the sister both patients deteriorated with pulmonary and hepatic involvement.
\end{abstract}

Shortly after the description of 'congenital agammaglobulinaemia' (Bruton, 1952) an 'acquired primary' form of the disease was recognised in adults (Grant and Wallace, 1954; Prasad and Koza, 1954; Prasad et al., 1957). Unlike the congenital variety there is no apparent pattern of inheritance but familial cases have been reported (Wollheim, 1961; Wollheim et al., 1964; Buckley and Sidbury, 1968). Multi-organ non-specific granulomata are a recognised feature of some cases (Rosen, 1971) and have been noted in a patient who also had depression of delayed-type hypersensitivity (Sharma and James, 1971). We are not aware of previous reports of this association in sibships.

We report acquired primary hypogammaglobulinaemia with widespread non-specific granulomata in a brother and sister, both of whom also had evidence of a defect in delayed-type hypersensitivity.

\section{Case reports}

CASE 1 The son of Maltese parents, domiciled in Great Britain for four years, presented at the age of 16 with iritis. He had also had purple lesions on both shins for six years. There were no other abnormal physical signs but a chest radiograph showed widespread mottled shadowing of both lungs with some ring shadows and bronchial wall thickening (Fig. 1). Routine haematology and blood biochemistry were normal but a 24-hour urinary calcium was raised at $\mathbf{9 \cdot 2} \mathrm{mmol} / 24$ hours (N 2.5-7.5). Sputum for acid-fast bacilli was negative on three occasions and Wasserman, Lepromin,
Kveim, and Mantoux tests (1/100) were negative (previous BCG vaccination aged 10). Biopsy of the skin lesion showed 'a large tuberculoid granuloma with one swollen and heavily infiltrated nerve. Very scanty acid-fast bacilli present'. Biopsies from nerve, liver, and unaffected skin revealed no histological abnormalities. He was treated with prednisolone and dapsone with improvement in the iritis but not in the pulmonary shadowing.

When aged 18 he was readmitted with severe measles associated with persistence of Koplik's spots, a right lower lobe pneumococcal pneumonia, and jaundice with a bilirubin of $130 \mu \mathrm{mol} /$ 1. After resolution of the pneumonia an open lung biopsy was performed. Histology showed many non-caseating granulomata in the lung and pleura (Fig. 2) with acid-fast bacilli present. He was treated with PAS, isoniazid, streptomycin, and prednisolone for three years but his condition continued to deteriorate.

$\mathrm{He}$ was readmitted on four separate occasions with moniliasis of the larynx, palmar warts, iritis, lobar pneumonia, pneumococcal meningitis, and fluctuating jaundice. Liver function tests showed a bilirubin rising to $265 \mu \mathrm{mol} / 1$, SGOT to 199 units (N8-35), and alkaline phosphatase to $75 \mathrm{KA}$ units (N4-14). Serum protein electrophoretic strips on two occasions demonstrated a moderate increase in $\alpha_{1}$ and $\alpha_{2}$ globulins with a decrease in $\gamma$ globulin. A liver biopsy showed two granulomata without caseation. The liver parenchymal cells were healthy and no acid-fast bacilli were seen. After antituberculosis chemotherapy had been stopped prednisolone, $7.5 \mathrm{mg}$ daily, was continued. 


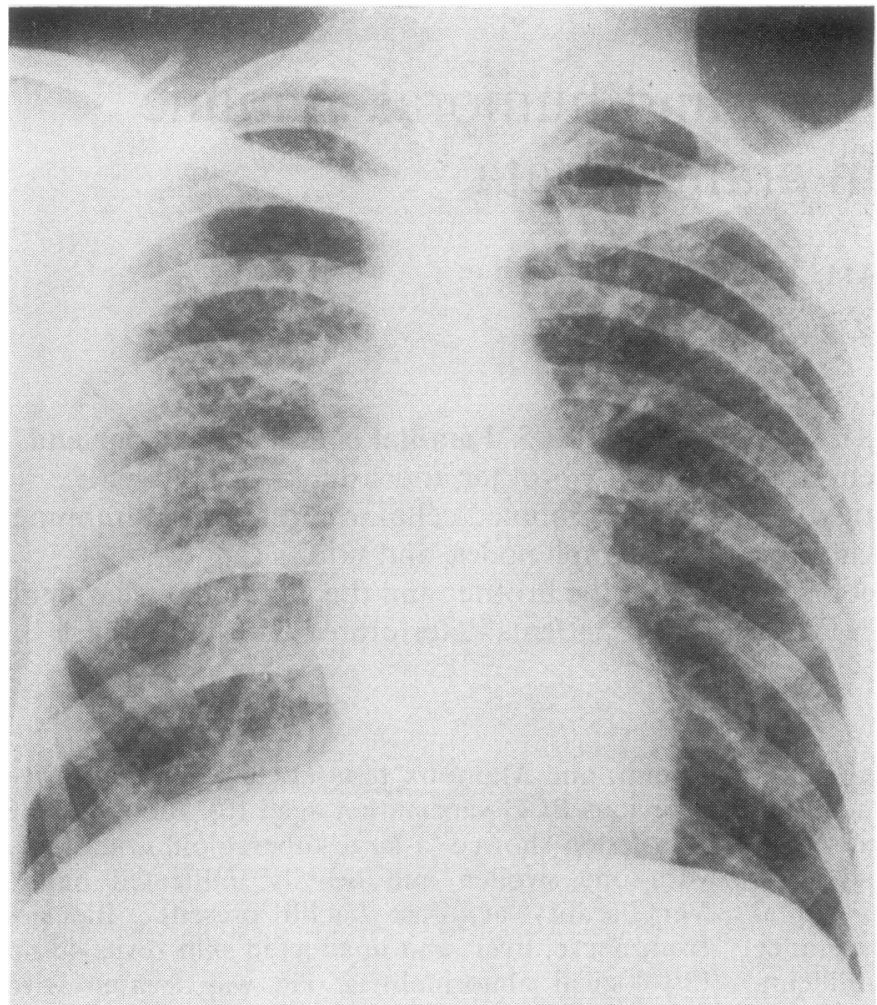

Fig. 1 Case 1. Chest radiograph showing widespread mottled shadowing of both lungs with some ring shadows and bronchial wall thickening.

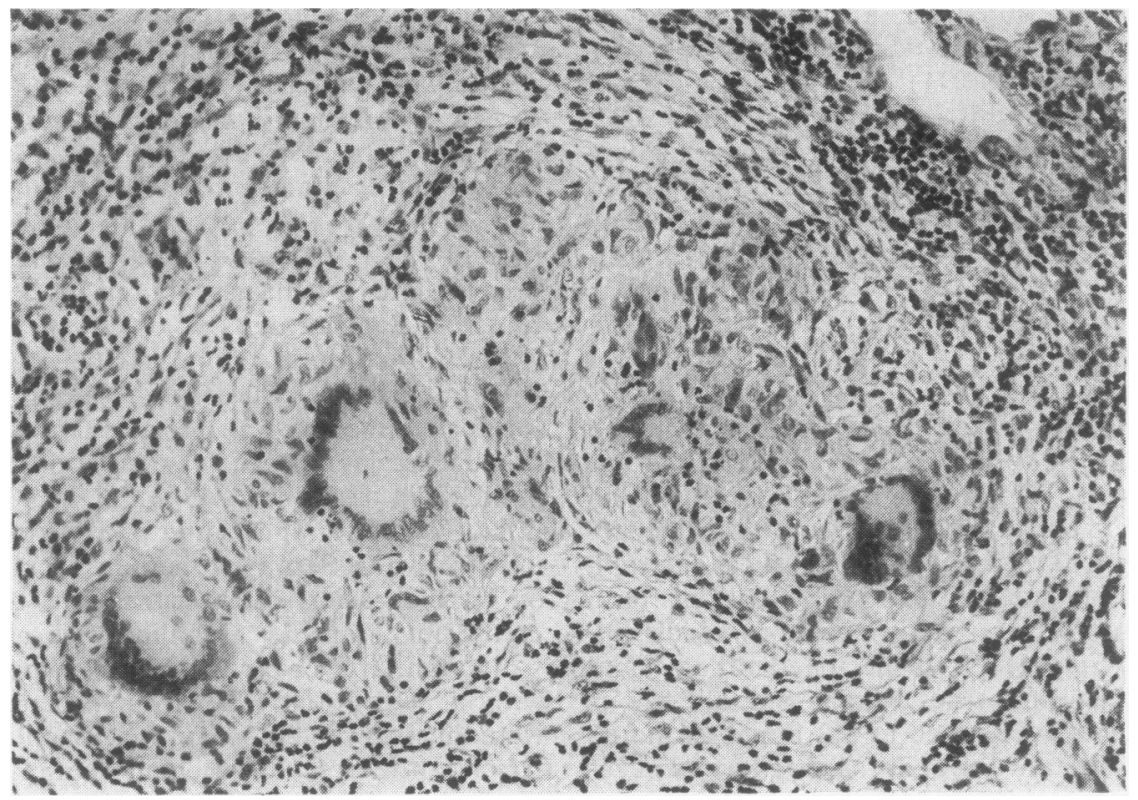

Fig. 2 Case 1. Lung biopsy showing several non-caseating granulomata. $H$ and $E \times 185$. 
At the age of 23 , because of a rising bilirubin and alkaline phosphatase, he underwent laparotomy. The gall bladder and common bile duct were dilated. There was a stricture of the terminal $1 \mathrm{~cm}$ of the common bile duct and the pancreas was indurated and oedematous. Sphincterotomy, cholecystoenterostomy, and wedge biopsy of liver were performed. Histology of the liver showed centrilobular bile retention, and in the portal tracts there was bile duct proliferation and chronic inflammatory cell infiltration. Numerous granulomata, negative for acid-fast bacilli, were present.

At the age of 25 he was admitted with a staphylococcal pneumonia. His jaundice deepened and he died eventually from a Gram-negative septicaemia. At no time during the patient's life was Mycobacterium tuberculosis cultured.

CASE 2 The sister of case 1 presented at the age of 25 with left pleuritic chest pain, cough, and copious sputum production. From early childhood she had suffered from recurrent sinus infections and persistent non-productive cough. Examination revealed bilateral basal crackles but neither clubbing nor hepatosplenomegaly. A chest radiograph showed 'increased reticular markings in both upper and lower zones and fairly extensive patchy consolidation in the left lower lobe with dilated bronchi'. A bronchogram showed bilateral basal bronchiectasis. Sputum for acid-fast bacilli and Mantoux (1/100) and Kveim tests were negative. A rectal biopsy was normal. Histology of a scalene node showed 'numerous follicular lesions with giant cells. The appearances are of sarcoidosis or tuberculosis without caseation. No acid-fast bacilli were seen'. Culture of the node was negative for tuberculosis. A diagnosis of sarcoidosis was made and treatment with intermittent antibiotics and postural drainage was begun.

At the age of 35 she was admitted to hospital with gradual worsening of dyspnoea and productive cough. She was cyanosed, clubbed, jaundiced, and emaciated. There were coarse crackles at both lung bases and hepatosplenomegaly. A chest radiograph showed widespread irregular mottled shadowing with numerous ring shadows and small linear shadows consistent with bronchiectasis (Fig. 3). The haemoglobin was $14.4 \mathrm{~g} / \mathrm{dl}$, white cell count $18.3 \times 10^{9} / 1$ (88\% neutrophils), and ESR $69 \mathrm{~mm}$ in 1 hour. There were no bacterial pathogens, acid-fast bacilli, aspergillus, or eosinophils in the sputum. Blood urea, electrolytes, and calcium were normal. The alkaline phosphatase was 372 IU (N 9-35), SGOT 48 IU (N 6-21), SGPT 57 IU (N 6-33), $\gamma$ GT 234 IU (N 9-29), and bilirubin $46 \mu \mathrm{mol} / 1$. Serum protein estimation was $67 \mathrm{~g} / \mathrm{l}$ (N 62-82), of which $36 \mathrm{~g} / 1$ was albumin and $31 \mathrm{~g} / 1$ globulin. Serum protein electrophoresis showed a

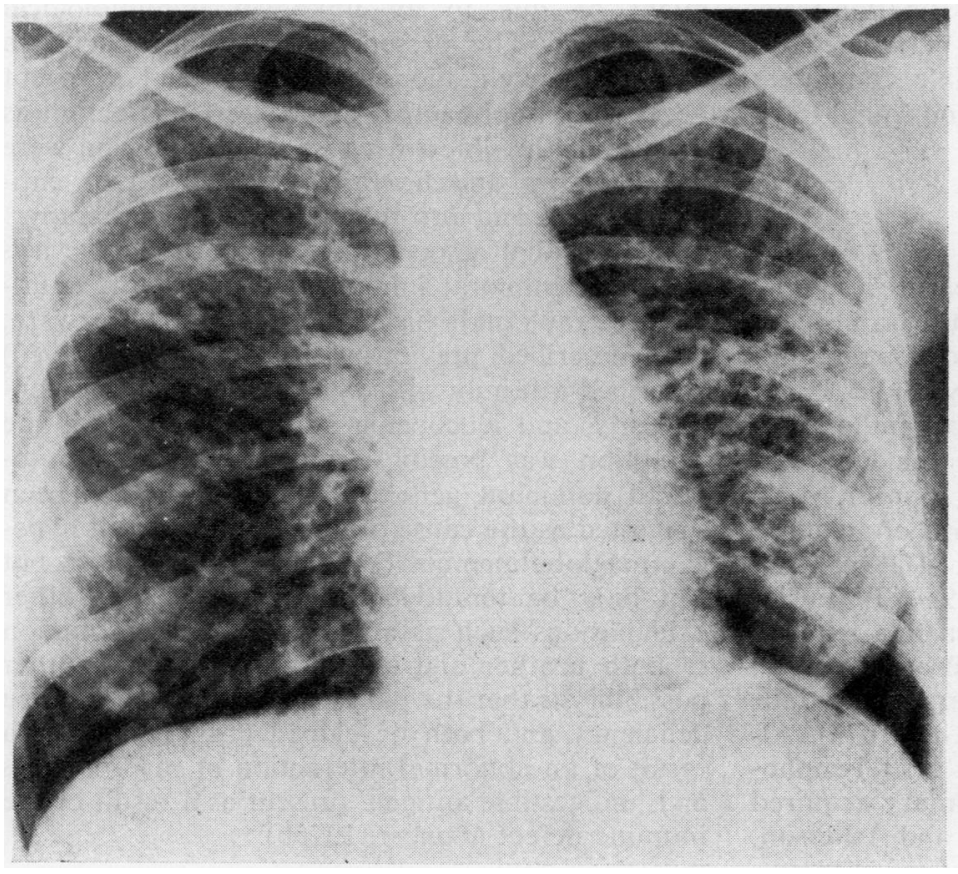

Fig. 3 Case 2. Chest radiograph showing widespread irregular mottled shadowing with numerous ring shadows and small linear shadows consistent with bronchiectasis. 
decreased $\gamma$ globulin. Immunoelectrophoresis revealed IgA less than 20 IU (N 57-172), IgM 39 IU (N 138-196), and IgG 111 IU (N 57-172). Antibodies to common bacterial and viral pathogens were absent, anti-B isohaemagglutinin titre was $1 / 4$, Escherichia coli antibody titre was $1 / 4$ $(\mathrm{N}>1 / 64)$, and a Mantoux (1/100) was negative (no previous BCG vaccination). Lymphocyte $T$ cell population was normal but lymphocyte transformation to phytohaemagglutinin (PHA) and purified protein derivative (PPD) was impaired. Lymphocyte 5-nucleotidase was $1.9 \mathrm{nmol} / \mathrm{h} / 10^{6}$ cells (N 15-35). Circulating phagocytosing monocytes were $27 \%$ (N 10-20\%). A Kveim biopsy was equivocal on two occasions. Serum angiotensin converting enzyme (ACE) was 37 units (N 15-30). A liver biopsy showed several small granulomata with giant cells. No acid-fast bacilli were seen. Lung function studies revealed a mixed restrictive and obstructive picture.

She was treated initially with conventional antituberculosis chemotherapy without improvement and then intramuscular human $\gamma$ globulin injections. One month after starting immunoglobulin replacement she was readmitted with worsening of breathlessness, deepening of jaundice, and right heart failure. This deterioration was improved by antibiotics and prednisolone at an initial dosage of $60 \mathrm{mg}$ per day. At the time of writing she is being treated with immunoglobulin injections and prednisolone, $10 \mathrm{mg}$ daily, with no symptomatic deterioration but worsening of liver function tests. Immunoglobulin estimations were performed on the mother and sister (the available remaining relatives of the patients described) and found to be within normal limits.

\section{Discussion}

The clinical and immunological features suggest that these patients suffered from the same condition. Both had gammaglobulin deficiency and immunoelectrophoresis and antibody studies in the sister showed low levels of immunoglobulin. This deficiency of gammaglobulin, with the low lymphocyte 5-nucleotidase in the sister (Johnson et al., 1977), is compatible with the diagnosis of acquired primary hypogammaglobulinaemia (Grant and Wallace, 1954; Prasad and Koza, 1954; Prasad et al., 1957). The hypogammaglobulinaemia is probably a result of a failure of the B-lymphocytes, which are present in normal numbers, to produce immunoglobulin (Gajl-Peczalska et al., 1973). Although there are normal numbers of T-lymphocytes in the peripheral blood in primary acquired hypogammaglobulinaemia (Webster and Asherson,
1974) in over half of the cases their function is im- $\frac{\overrightarrow{3}}{\frac{3}{2}}$ paired (Douglas et al., 1970) and this feature was $\frac{5}{5}$ demonstrated in the sister. A T-lymphocyte defect $\frac{\bar{c}}{\bar{c}}$ was probably present in the brother in view of the $\frac{\vec{\sigma}}{}$ negative tuberculin reactivity and recurrent viral $\varrho$ and fungal infections. This is of interest because $\tilde{\infty}$ patients with primary acquired hypogammaglobu- $\overrightarrow{0}$ linaemia, as opposed to combined immune $\overrightarrow{-}$ deficiency, do not suffer from viral or fungal infec- $\vec{\omega}$ tions, probably because there are functional $\mathrm{T} \stackrel{\text { }}{\vec{P}}$ lymphocytes present (Webster and Asherson, 1974). $\stackrel{x}{x}$

Our patients were shown to have multi-organ $\omega_{\omega}^{\omega}$ granulomata and this was associated with one episode each of iritis and hypercalciuria in the $\overrightarrow{0}$ brother and a raised serum ACE in the sister (Lie- $\overrightarrow{0}$ berman, 1975). Both could, therefore, be con- $\frac{9}{I}$ sidered to have sarcoidosis. Against this diagnosis $\vec{T}$ are the atypical clinical and immunological fea- $\mathbb{D}$ tures (Mitchell and Scadding, 1974) and the equivocal or negative Kveim biopsies in the presence of active granulomata (Sharma and James, 1971). Neither multi-system granulomata $\vec{\bullet}$ (Lancet, 1975a) nor the raised ACE are specific to 9 sarcoidosis. The latter has been described in Gaucher's disease (Liebermann and Beutler, 1976) and in hypogammaglobulinaemia with multisystem granulomata (Turton, 1977, personal com- ڤ munication). Sharma and James (1971) reported a $\stackrel{\unrhd}{\unrhd}$ similar case to those we have described and con- $\overrightarrow{\vec{A}}$ cluded that their patient did not have sarcoidosis $\frac{9}{3}$ and that the associated granulomatous response might be due to an unknown immunological mechanism. The presence of acid-fast bacilli in two out of eight biopsies in our case 1 does not necessarily imply active myobacterial infection as $\underset{\times}{\stackrel{\varpi}{~}}$ a cause of the illness because on no occasion were $\frac{0}{0}$ bacilli grown and he failed to respond to anti- 3 . lepromatous and anti-tuberculosis chemotherapy.

The familial association of a defect in the cellular and humoral immune response with multi- $ᄋ$ organ granulomata has not, as far as we are aware, been described previously. Kushner et al. (1960)을. described a family with hypoglobulinaemia, splenomegaly, and leucopenia and concluded that the ? condition was possibly transmitted by an auto- $N$ somal dominant gene. A genetic basis has been $\underset{\mathrm{W}}{\mathrm{W}}$ suggested as the cause of primary 'acquired' hypo- 0 gammaglobulinaemia (Wolheim et al., 1964) but can only be tentative in our patients as other $\frac{D}{D}$ explanations such as an infective agent common $\stackrel{\oplus}{\rightarrow}$ to both brother and sister are possible. Another 0 possibility is that the patients had familial immune deficiency and both developed granulomata as a $\stackrel{\mathbb{D}}{\Omega}$ result of an abnormal interaction of macrophages $\mathbb{\mathbb { D }}$ and 'indigestible antigen' present as a result of the immune defect (Lancet, 1975b). 
The treatment of both patients has been disappointing. Steroids and antituberculosis therapy did not halt the clinical deterioration and eventual death of case 1 and the addition of immunoglobulin injections in case 2 has produced no clinical benefit. We feel that further understanding of this unusual condition is essential to make treatment rational.

We thank Drs. E. E. Keal and P. D. Heaf for permission to study patients under their care, Drs. A. D. B. Webster and P. Cole for the immunological investigations and advice, and Dr. B. Heard for assistance with the histology.

\section{References}

Bruton, O. C. (1952). Agammaglobulinaemia. Paediatrics, 9, 722-727.

Buckley, R. M., and Sidbury, J. B., Jr. (1968). Hereditory alterations in the immune response: coexistance of agammaglobulinaemia, acquired hypogammaglobulinaemia and selective immunoglobulin deficiency in a sibship. Paediatric Research, 2, 72-84.

Douglas, S. D., Goldberg, L. S., and Fudenberg, H. H. (1970). Clinical, serological and leukocyte function studies on patients with idiopathic 'acquired' agammaglobulinaemia and their families. American Journal of Medicine, 48, 48-53.

Gajl-Peczalska, K. J., Park, B. H., Biggar, W. D., and Good, R. A. (1973). B and T lymphocytes in primary immunodeficiency disease in man. Journal of Clinical Investigation, 52, 919-928.

Grant, G. H., and Wallace, W. D. (1954). Agammaglobulinaemia. Lancet, 2, 671-673.

Johnson, S. M., North, M. E., Asherson, G. L., Allsop, J., Watts R. W. E., and Webster, A. D. B. (1977). Lymphocyte purine 5-nucleotidase deficiency in primary hypogammaglobulinaemia. Lancet, 1, 168-170.

Kushner, D. S., Dubin, A., Donlon, W. P., and Bronsky, D. (1960). Familial hypogammaglobulinaemia, splenomegaly and leukopenia: with a review of the etiological factors of the hypogam- maglobulinemias. American Journal of Medicine, 29, 33-42.

Lancet (1975a). Granulomas of the liver (Editorial). Lancet, 2, 1079-1080.

Lancet (1975b). Form and function of the sarcoid granuloma (Editorial). Lancet, 2, 1292.

Lieberman, J. (1975). Elevation of serum angiotensinconverting-enzyme (ACE) level in sarcoidosis. American Journal of Medicine, 59, 365-372.

Lieberman, J., and Beutler, E. (1976). Elevation of serum angiotensin-converting-enzyme in Gaucher's disease. New England Journal of Medicine, 294, 1442-1444.

Mitchell, D. N., and Scadding, J. G. (1974). Sarcoidosis. American Review of Respiratory Disease, 110, 774-802.

Prasad, A. S., and Koza, D. W. (1954). Agammaglobulinaemia. Annals of Internal Medicine, 41, 629639.

Prasad, A. S., Reiner, E., and Watson, C. J. (1957). Syndrome of hypogammaglobulinaemia, splenomegaly and hypersplenism. Blood, 12, 926-932.

Rosen, F. S. (1971). The thymus gland and immunedeficiency syndromes. In Immunological Diseases, edited by M. Samter, p. 504. Little, Brown, Boston.

Sharma, O. P., and James, D. G. (1971). Hypogammaglobulinemia, depression of delayed-type hypersensitivity and granuloma formation. American $R e$ view of Respiratory Disease, 104, 228-231.

Webster, A. D. B., and Asherson, G. L. (1974). Identification and function of $T$ cells in the peripheral blood of patients with hypogammaglobulinaemia. Clinical and Experimental Immunology, 18, 499-504.

Wollheim, F. A. (1961). Inherited 'acquired' hypogammaglobulinaemia. Lancet, 1, 316-317.

Wollheim, F. A., Belfrage, S., Cöster, C., and Lindholm, H. (1964). Primary 'acquired' hypogammaglobulinaemia, clinical and genetic aspects of nine cases. Acta Medica Scandinavica, 176, 1-16.

Requests for reprints to: Dr. W. H. Perks, Brompton Hospital, Fulham Road, London SW3 6HP, UK. 